\title{
A model for the radio-continuum emission from the P Cygni wind
}

\author{
R. F. González ${ }^{1}$ and J. Cantó ${ }^{2}$ \\ ${ }^{1}$ Centro de Radioastronomía y Astrofísica, UNAM, Morelia Mich., México \\ e-mail: rf.gonzalez@astrosmo.unam.mx \\ 2 Instituto de Astronomía, UNAM, México D.F., México
}

Received 6 March 2007 / Accepted 6 November 2007

\section{ABSTRACT}

\begin{abstract}
Here we present a simple, semianalytic model for estimating the free-free radiation (at radio wavelengths) from the P Cygni stellar wind. Recent observations from this source at different epochs show time-dependent flux densities and spectral indices, which clearly deviate from the standard models of ionized extended envelopes around massive stars. In this work, we study the contribution by shocks to the total emission from a completely ionized stellar wind, which results from variations in the wind parameters at injection. In particular, we consider sudden changes in the wind velocity and mass loss rate that instantaneously produce two-shock wave structures (called working surfaces) that travel down the flow. The compressed layer between the two shocks emits continuum radiation that can be detected in the radio band. We show that our model gives a possible explanation for the observed variability in the flux densities and spectral indices from the P Cygni wind.
\end{abstract}

Key words. hydrodynamics - radio continuum: ISM - stars: individual: P Cygni

\section{Introduction}

The massive B1 Ia star P Cyg is one of the most luminous stars in our galaxy. Located at a distance of $1.7 \mathrm{kpc}$ (Najarro et al. 1997), it is the nearest luminous blue variable (LBV) star. The LBVs stars are extremely massive hypergiants, which have circumstellar envelopes formed by mass loss in strong stellar winds (e.g. Skinner et al. 1998, and references therein). These stars are very unstable and suffer violent episodes of heavy mass loss. P Cyg is one of the two LBVs in our galaxy which have been observed during a giant eruption: the P Cyg eruptive event in the 1600s and the $\eta$ Car great eruption in the 1840s (e.g. Humphreys et al. 1999). As a result of the $\eta$ Car eruptive event, an optical bipolar nebula known as the "Homunculus" was formed, extending $\pm 8^{\prime \prime}$ along its major axis, and probably the P Cyg eruption in the 1600 s produced the observed (in infrared emission lines) $\sim 10^{\prime \prime}$ nebula around the star (see Smith \& Hartigan 2006).

At radio frequencies, $\mathrm{P}$ Cyg has also been observed repeatedly (e.g. Abbott et al. 1981; Contreras et al. 1996, 2004; Skinner et al. 1998; Exter et al. 2002). It is a very strong source with a radio-continuum spectrum consistent with thermal emission, but the spectral indices obtained from the observed flux densities deviate from the expected 0.6 value for an isotropic wind (Panagia \& Felli 1975; Wright \& Barlow 1975). In addition, it clearly shows variability in its flux densities which has been previously attributed to changes in the degree of ionization (van den Oord et al. 1985), but could also be related to variations in the wind parameters (e.g. Scuderi et al. 1994). Observations by Skinner et al. (1998) at 2 and $6 \mathrm{~cm}$ show that P Cyg is surrounded by an inhomogeneous nebula which extends to almost one arcminute from the star. The radio nebula consists of a compact core of a few tenths of an arcsecond in diameter, and an inner nebula with a diameter of roughly $18^{\prime \prime}$ (which probably corresponds to the outburst during the 17th Century). They found that the radio emission from P Cyg is variable on time-scales no longer than one month. Contreras et al. (1996) made multifrequency radio-continuum observations to derive mass loss rates for massive stars. In particular, the P Cyg flux densities vary by $\sim 50 \%$ over a period of $\sim 1 \mathrm{yr}$ and the observed values cannot be explained by previous models of the variability (ionization degree, wind velocity, mass loss rate). In Contreras et al. (2004), it is confirmed that P Cyg is a variable source both in its radio flux density and morphology. Comparing the new fluxes with those reported in Contreras et al. (1996), they found that they have considerably decreased $(\sim 70 \%, 35 \%$ and $34 \%$ at $0.7,3.6$ and $6 \mathrm{~cm}$, respectively).

As pointed out by Raga et al. (1990), supersonic flows are always subject to the development of shock waves. They show that supersonic variabilities in the ejection velocity result in the formation of two-shock wave structures (called working surfaces) that travel down the flow. Between the shocks a layer of compressed material grows due to the continuous feeding of shocked stellar winds. Ghavamian \& Hartigan (1998) computed the continuum emission at radio wavelengths from interstellar shock waves, showing that they emit a sizeable amount of radiation. More recently, González \& Cantó (2002) developed a model to calculate the radio-continuum emission from shocked stellar winds in low mass stars. In their model (see also González et al. 2006), they propose that the emission is produced by internal working surfaces forming in the wind as a result of variabilities in the wind parameters at injection. Specifically, they proposed that the emission is produced in the cooling region behind the shocks where the flow remains collisionally ionized. The compressed layer between the shocks does not contribute to the emission, since it is mostly neutral, as a result of an insufficient stellar flux of UV photons to ionize either this region or the wind itself.

In the present work, we will consider the opposite situation, namely, that the star emits enough UV radiation to keep both the wind and the compressed layer inside the working surface fully ionized. Furthermore, we will ignore the contribution of the cooling regions to the emission of the working surface and 


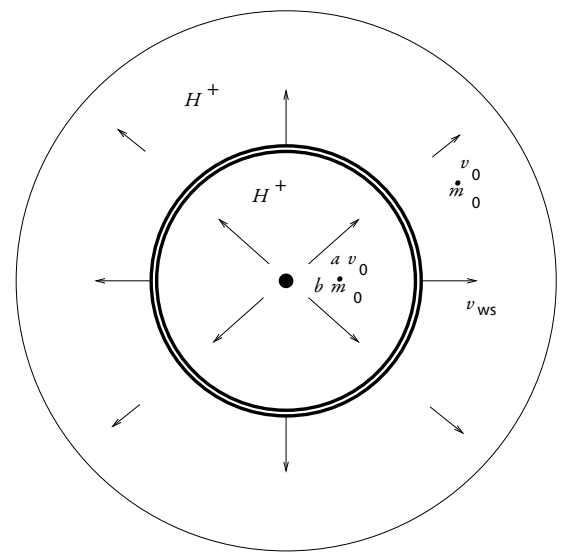

Fig. 1. Schematic diagram showing a single working surface (which is bounded by two shocks; thick lines) produced in a photoionized stellar wind. The wind, that is assumed to be isotropic, is initially ejected with velocity $v_{0}$ and mass loss rate $\dot{m}_{0}$, which suddenly change to $a v_{0}$ and $b \dot{m}_{0}$ (with $a>1, b>1$ or $\leq 1$ ), respectively. The working surface moves with an intermediate velocity $v_{\mathrm{ws}}$.

assume that it comes from the compressed layer. Supposing that the wind parameters (ejection velocity and mass loss rate) are suddenly increased gives as a result the formation of a working surface, whose kinematical properties (velocity and position) can be obtained from the formalism developed by Cantó et al. (2000). Then, we compute the optical depth of the working surface and the total optical depth along each line of sight in order to obtain the emission from the whole configuration. We show that our model of a photoionized wind with internal shocks gives a possible explanation for the variation in the flux densities observed in the P Cyg stellar wind.

The paper is organised as follows. In Sect. 2, we describe the model and present our predictions. Section 3 shows the comparison of our model results with the radio-continuum observations of P Cyg stellar wind. Finally, in Sect. 4 we give our conclusions.

\section{The model}

We consider a spherical stellar wind which is completely ionized from a central star of radius $r_{0}$. For times $t<0$, the wind velocity and the mass loss rate are constant with values $v_{0}$ and $\dot{m}_{0}$, respectively. At time $t=0$, the ejection velocity is drastically increased to $a v_{0}(a>1)$, while the mass loss rate is suddenly changed to $b_{0} \dot{m}_{0}(b>1$ or $b \leq 1)$. Such variation in the flow parameters forms instantaneously (at the base of the wind) one working surface that moves with a constant velocity $v_{\mathrm{ws}}$ intermediate between the low-velocity downstream flow and the faster upstream wind (see Cantó et al. 2000; González \& Cantó 2002). The inner shock decelerates the fast upstream wind, while the external shock accelerates the downstream low-velocity wind up to the velocity $v_{\mathrm{ws}}$ of the working surface (Fig. 1).

From the formalism presented in Cantó et al. (2000), it can be shown (see also Cantó et al. 2005; González et al. 2006) that,

$v_{\mathrm{ws}}=\sigma v_{0}$,

with $\sigma=\left(a^{1 / 2}+a b^{1 / 2}\right) /\left(a^{1 / 2}+b^{1 / 2}\right)$. For constant mass loss rate $(b=1)$, the velocity of the working surface is simply $v_{\mathrm{ws}}=a^{1 / 2} v_{0}$.

Since $v_{\mathrm{ws}}$ is constant, the position of the working surface $r_{\mathrm{ws}}$ from the central star after a time $t$ is obtained by,

$r_{\mathrm{ws}}=r_{0}+v_{\mathrm{ws}} t$.
(2) $\left(\frac{v}{v_{\mathrm{c}}}\right)^{-2.1}=\frac{\chi_{v}}{r_{\mathrm{ws}}^{3}}\left(\frac{\dot{m}_{0}}{4 \pi \bar{m} v_{0}}\right)^{2}$.

Between the shocks a shell of shocked winds is formed, growing in mass with time. Given the position (Eq. (2)) and velocity (Eq. (1)) of the working surface, it is possible to calculate the contribution of this shell to the optical depth along a line of sight intersecting the working surface.

At a time $t$, the incorporated mass from the upstream and downstream winds into the working surface is given by,

$m_{\mathrm{ws}}=\beta \dot{m}_{0} t$,

where $\beta=\sigma-1+(b / a)(a-\sigma)$.

Assuming strong isothermal shocks, the pressure inside the working surface is $P_{\mathrm{ws}}=\rho v_{\mathrm{s}}^{2}$ where $\rho$ is the pre-shock density and $v_{\mathrm{s}}$ is the shock velocity. This pressure is given by,

$$
\begin{aligned}
P_{\mathrm{ws}} & =\frac{\dot{m}_{0}}{4 \pi r_{\mathrm{ws}}^{2} v_{0}}\left(v_{\mathrm{ws}}-v_{0}\right)^{2} \\
& =\frac{b \dot{m}_{0}}{4 \pi r_{\mathrm{ws}}^{2} a v_{0}}\left(a v_{0}-v_{\mathrm{ws}}\right)^{2}
\end{aligned}
$$

which is consistent with Eq. (1), since $v_{\mathrm{ws}}$ is constant in time in our model. The density inside the working surface is given by $\rho_{\mathrm{ws}}=P_{\mathrm{ws}} / c_{\mathrm{s}}^{2}$ where $c_{\mathrm{s}}$ is its isothermal sound speed (e.g. Dyson $\&$ Williams 1980). Then,

$\rho_{\mathrm{ws}}=\frac{\dot{m}_{0}}{4 \pi v_{0} c_{\mathrm{s}}^{2}}\left(\frac{\sigma-1}{\sigma}\right)^{2} \frac{1}{t^{2}}$,

where we have assumed $r_{0} \ll v_{\text {ws }} t$ in Eq. (2).

The mass $m_{\mathrm{ws}}$ (Eq. (3)) must be equal to the mass $\left(=4 \pi r_{\mathrm{ws}}^{2} \rho_{\mathrm{ws}} \delta r_{\mathrm{ws}}\right)$ contained in the spherical shell of thickness $\delta r_{\mathrm{ws}}$. Therefore,

$\delta r_{\mathrm{ws}}=\frac{\beta c_{\mathrm{s}}^{2}}{(\sigma-1)^{2} v_{0}} t$.

From Eqs. (4) and (5), we are able to compute the emission measure $\epsilon_{\mathrm{ws}}\left(=\left[\rho_{\mathrm{ws}} / \bar{m}\right]^{2} \delta r_{\mathrm{ws}}\right)$ of the working surface,

$\epsilon_{\mathrm{ws}}=\left(\frac{\dot{m}_{0}}{4 \pi \bar{m} c_{\mathrm{s}}}\right)^{2} \frac{(\sigma-1)^{2} \beta}{\sigma r_{\mathrm{ws}}^{3}}$,

and, finally, its optical depth $\tau_{\mathrm{ws}}\left(=\epsilon_{\mathrm{ws}} \chi_{v}\right.$, with $\chi_{v}=8.436 \times$ intersecting the central star. For a line of sight intersecting the working surface at an angle $\theta\left(=\sin ^{-1}\left[q / r_{\mathrm{ws}}\right]\right.$ with $q$ the impact parameter; see Fig. 2) from the normal, the optical depth of the shell is given by,

$\tau_{\mathrm{ws}}(\tilde{q})=\left(\frac{v}{v_{\mathrm{c}}}\right)^{-2.1} I_{\mathrm{ws}}(\tilde{q})$,

with

$I_{\mathrm{ws}}(\tilde{q})=2\left(\frac{v_{0}}{c_{\mathrm{s}}}\right)^{2} \frac{\beta(\sigma-1)^{2}}{\sigma} \frac{1}{\left(1-\tilde{q}^{2}\right)^{1 / 2}}$,

where the factor 2 takes into account the fact that any line of sight intersects both the approaching and receding sides of the working surface, and we have defined the dimensionless parameter $\tilde{q}=q / r_{\mathrm{ws}}$, and a critical frequency $v_{\mathrm{c}}$ such that, $10^{-7} v^{-2.1}$, being $v$ the frequency in $\mathrm{Hz}$ ) along a line of sight 
In order to calculate the radio-continuum emission at a time $t$ from the whole system, it is necessary to compute the total optical depth $\tau(\tilde{q})$ along each line of sight. According to Panagia \& Felli (1975; see also Wright \& Barlow 1975), the optical depth along a line of sight with $\tilde{q}>1$ (which has only the contribution by the external wind) is calculated by,

$\tau(\tilde{q})=2 \int_{0}^{\infty} n^{2}(s) \chi_{\nu} \mathrm{d} s=\frac{\pi}{2 \tilde{q}^{3}}\left(\frac{v}{\nu_{\mathrm{c}}}\right)^{-2.1}$.

Otherwise, the optical depth along a line of sight with $\tilde{q} \leq 1$ has the contribution of both the fast and slow winds and also of the working surface. The optical depth along the line of sight in this case is given by,

$\tau(\tilde{q})=\tau_{\mathrm{ew}}(\tilde{q})+\tau_{\mathrm{ws}}(\tilde{q})+\tau_{\mathrm{iw}}(\tilde{q})$,

where $\tau_{\text {ws }}(\tilde{q})$ is given by Eq. (7), and the optical depths of the downstream and the upstream winds are,

$\tau_{\text {ew }}(\tilde{q})=2 \int_{\left(1-\tilde{q}^{2}\right)^{1 / 2}}^{\infty} n^{2}(s) \chi_{\nu} \mathrm{d} s$

and

$\tau_{\mathrm{iw}}(\tilde{q})=2 \int_{0}^{\left(1-\tilde{q}^{2}\right)^{1 / 2}} n^{2}(s) \chi_{\nu} \mathrm{d} s$,

respectively. From Panagia \& Felli (1975) and Wright \& Barlow (1975), it can be shown that,

$\tau_{\mathrm{ew}}(\tilde{q})=\left(\frac{v}{\nu_{\mathrm{c}}}\right)^{-2.1} I_{\mathrm{ew}}(\tilde{q})$

and, analogously,

$\tau_{\mathrm{iw}}(\tilde{q})=\left(\frac{v}{v_{\mathrm{c}}}\right)^{-2.1} I_{\mathrm{iw}}(\tilde{q})$,

where,

$I_{\mathrm{ew}}(\tilde{q})=\frac{\pi}{2 \tilde{q}^{3}}-\frac{\tilde{q}\left(1-\tilde{q}^{2}\right)^{1 / 2}+\operatorname{arctg}\left(\left[\left(1-\tilde{q}^{2}\right)^{1 / 2}\right] / q\right)}{\tilde{q}^{3}}$,

and,

$I_{\mathrm{iw}}(\tilde{q})=\left(\frac{b}{a}\right)^{2}\left[\frac{\tilde{q}\left(1-\tilde{q}^{2}\right)^{1 / 2}+\operatorname{arctg}\left(\left[\left(1-\tilde{q}^{2}\right)^{1 / 2}\right] / q\right)}{\tilde{q}^{3}}\right]$.

We have assumed that the system is far enough $\left(r_{0} \ll D\right)$ so that the lines of sights intersecting the central star can be ignored. Then, we estimated the intensity emerging from each direction and calculated the flux density by integrating the intensity over the solid angle.

The radio-continuum flux from the shocked stellar wind located at a distance $\mathrm{D}$ from the observer is then given by,

$S_{v}=2 \pi B_{v}\left(\frac{r_{\mathrm{ws}}}{D}\right)^{2} \int_{0}^{\infty}\left(1-\mathrm{e}^{-\tau(\tilde{q})}\right) \tilde{q} \mathrm{~d} \tilde{q}$,

where $B_{v}$ is the Planck function in the Rayleigh-Jeans approximation ( $=2 k T_{\mathrm{e}} v^{2} / c^{2}$ being $k$ the Boltzmann's constant, $T_{\mathrm{e}}$ the electron temperature and $c$ the light speed).

Defining the parameter $f_{\mathrm{c}}=\pi k T_{\mathrm{e}}\left(2 r_{\mathrm{ws}} / c D\right)^{2} v_{\mathrm{c}}^{2}$, we finally obtain,

$S_{v}=f_{\mathrm{c}}\left(\frac{v}{\nu_{\mathrm{c}}}\right)^{2} \int_{0}^{\infty}\left(1-\mathrm{e}^{-\tau(\tilde{q})}\right) \tilde{q} \mathrm{~d} \tilde{q}$

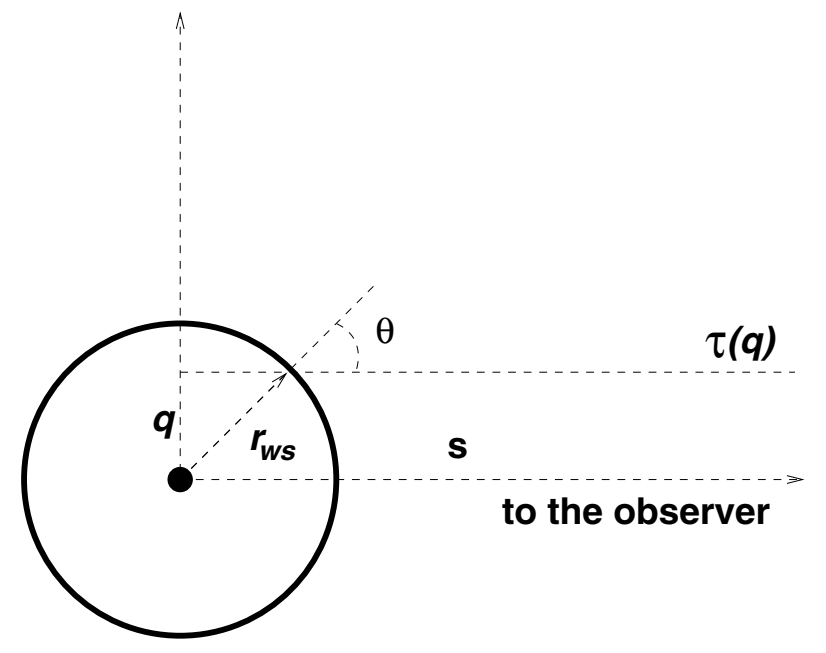

Fig. 2. Schematic diagram showing the adopted frame of reference for calculating the optical depth $\tau(q)$ along a given line of sight. For $q \leq r_{\mathrm{ws}}$ the line of sight intersects the spherical shell at an angle $\theta$ from the normal.

where,

$f_{\mathrm{c}}=\frac{4 \pi k T_{\mathrm{e}}}{c^{2}}\left(\frac{r_{\mathrm{ws}}}{D}\right)^{2} v_{\mathrm{c}}^{2}$

and $\tau(\tilde{q})$ is given by Eqs. (9) and (10). Equation (14) represents an autosimilar solution for the free-free radiation from a completely ionized stellar wind with time-dependent injection parameters. Note that the the critical frequency $v_{\mathrm{c}} \propto t^{-1.43}$ (Eq. (8)) and $f_{\mathrm{c}} \propto t^{-0.86}$ (Eq. (15)). In the particular case with $a=1$ and $b=1$, it can be shown from Eqs. (8), (9), (14) and (15) that the free-free radiation from the stellar wind $S_{v} \propto v^{0.6}$.

We are now in position to calculate the flux density from a photoionized stellar wind with internal shocks resulting from variations in the injection parameters. We have to specify the distance $D$ from the observer, the terminal velocity $v_{0}$ and the mass loss rate $\dot{m}_{0}$ together with the jump factors $a$ and $b$. Figures 3 and 4 show some examples.

In Fig. 3, we present two models for the emission from a completely ionized stellar wind with time dependent injection parameters. In both models, we have choosen representative parameters for the P Cyg stellar wind. A stellar wind with an initial ejection velocity $v_{0}=220 \mathrm{~km} \mathrm{~s}^{-1}$ that is suddenly increased to $330 \mathrm{~km} \mathrm{~s}^{-1}(a=1.5)$. In the top panel, we also assumed a constant mass loss rate $\dot{m}_{0}=10^{-5} M_{\odot} \mathrm{yr}^{-1}(b=1)$. From these values we obtain from Eq. (1) a working surface velocity $v_{\mathrm{ws}}=269.4 \mathrm{~km} \mathrm{~s}^{-1}(\sigma=1.2)$. It is shown that at low-frequencies $\left(v \ll v_{\mathrm{c}}\right.$ ) (where the system is optically thick; see Eqs. (7), (11) and (12)) the flux density approaches the emission from an isotropic stellar wind (with velocity $v_{0}$ ) predicted by Panagia \& Felli (1975) and Wright \& Barlow (1975), that is $S_{v} \propto v^{0.6}$. At high frequencies $\left(v \gg v_{\mathrm{c}}\right.$ where the system becomes optically thin and we are able to see the contribution of the upstream wind) the flux again approaches the spectral index $\sim 0.6$ but corresponding to a faster wind with velocity $a v_{0}$. Near the critical frequency $\left(v \simeq v_{\mathrm{c}}\right.$ ), there is the contribution of the working surface to the total emission. The flux is increased by the emission of the shell and a kind of a "bump" is observed. In time, this bump moves towards lower frequencies $\left(v \propto t^{-1.43}\right)$, while decreasing its intensity $\left(f_{\mathrm{c}} \propto t^{-0.86}\right)$. For the model presented in the bottom panel we supposed that the mass loss rate is increased by a factor $b=2$. As it is expected, the model presented in the 

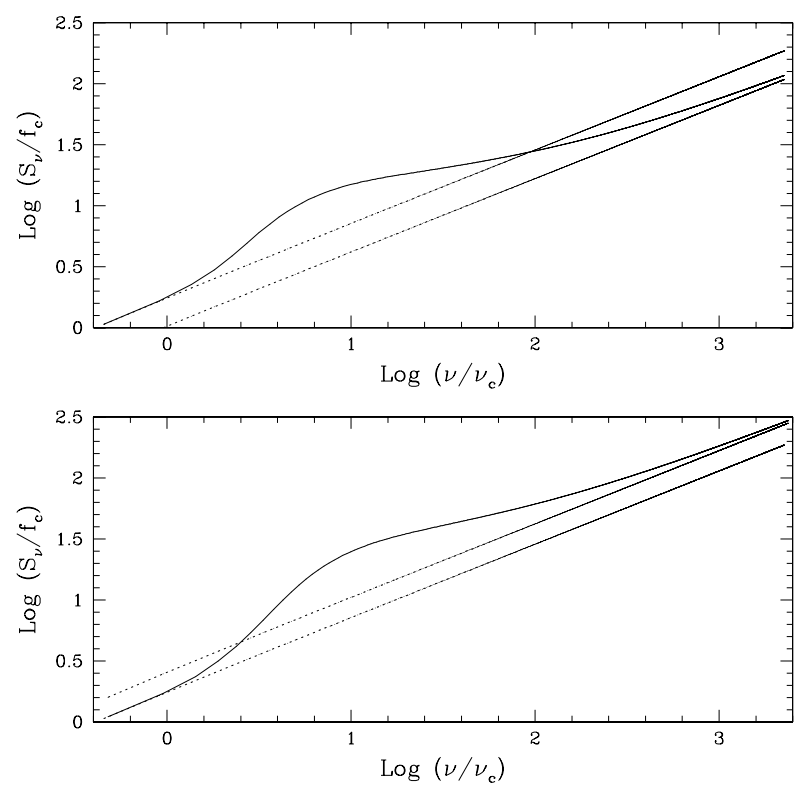

Fig. 3. Predicted free-free emission for two different models from a completely ionized and isotropic stellar wind with an internal working surface. We have adopted in both models an initial wind velocity $v_{0}=220 \mathrm{~km} \mathrm{~s}^{-1}$ which suddenly increased to $330 \mathrm{~km} \mathrm{~s}^{-1}(a=1.5)$. The mass loss rate $\dot{m}_{0}=10^{-5} M_{\odot} \mathrm{yr}^{-1}$ is assumed to be constant $(b=1$; top panel), and changed to $2 \times 10^{-5} M_{\odot} \mathrm{yr}^{-1}(b=2$; bottom panel). In both panels, the dashed lines represent the fluxes $\left(\propto v^{0.6}\right)$ from the stellar winds. Top panel: $v_{0}$ (upper line) and $a v_{0}$ (lower line); Bottom panel: $v_{0}$ (lower line) and $a v_{0}$ (upper line). The behaviour of the plots is described in the text.

bottom panel shows the same behaviour at $v \ll v_{\mathrm{c}}$ as that observed in the top panel. Nevertheless the emission at $v \geq v_{\mathrm{c}}$ from the shell and the upstream wind (with $b \dot{m}_{0}=2 \times 10^{-5} M_{\odot} \mathrm{yr}^{-1}$; $b=2$ ) is more important.

In Fig. 4, we present the radio-continuum flux for different values of the velocity increase factor $a$. In our models, we have assumed an initial stellar wind with $v_{0}=220 \mathrm{~km} \mathrm{~s}^{-1}$ that drastically changes by factors $a=1.3,1.5$, and 1.7. Additionally, we suppose that the mass loss rate $\dot{m}=10^{-5} M_{\odot} \mathrm{yr}^{-1}$ is constant $(b=1)$. With these parameters, the working surface velocities (Eq. (1)) are $v_{\mathrm{ws}}=250.8,269.4$, and $286.8 \mathrm{~km} \mathrm{~s}^{-1}$, respectively. We note that as $a$ increases the emission from shocks (at $v \simeq v_{\mathrm{c}}$ ) becomes more important and the contribution by the working surface extends to a wider range of frequencies. As mentioned above, at low frequencies $\left(v \ll v_{\mathrm{c}}\right)$, the flux is optically thick and in all models it approaches the emission $\left(\propto v^{0.6}\right)$ from the wind with velocity $v_{0}$. In the optically thin regimen (at high frequencies with $v \gg v_{\mathrm{c}}$ ), however, the flux decreases with $a$ and approaches the emission from the faster wind with velocity $a v_{0}$. Figures 3 and 4 show how sensitive our model is to the change parameters $a$ and $b$.

\section{Predicted radio-continuum emission from P Cygni}

As mentioned in Sect. 1, observations at radio frequencies by different authors (e.g. Abott et al. 1981; Contreras et al. 1996, 2004; Skinner et al. 1998) show that P Cyg is a very bright radio-continuum source. The observed spectrum is consistent with free-free emission, but clearly deviates from the predicted spectrum $\left(S_{v} \propto v^{0.6}\right)$ by Panagia \& Felli (1975) and Wright $\&$ Barlow (1975) for an isotropic stellar wind photoionized by

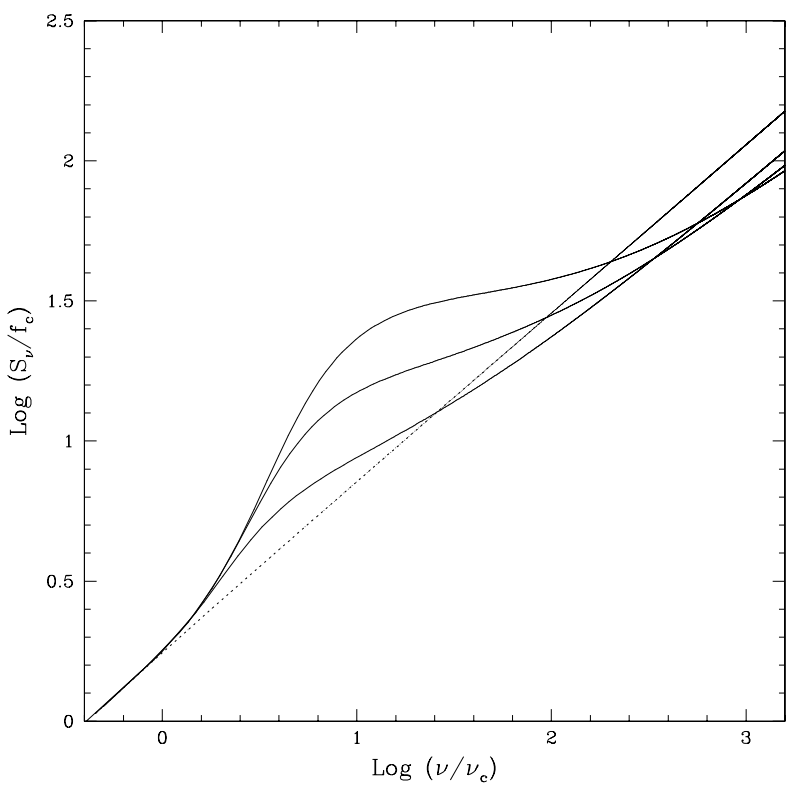

Fig. 4. Predicted free-free emission from an isotropic stellar wind with an internal working surface. The wind is initially ejected with $v_{0}=$ $220 \mathrm{~km} \mathrm{~s}^{-1}$ which is drastically changed by the increase factors $a=$ $1.3,1.5$, and 1.7. The mass loss rate $\dot{m}_{0}=10^{-5} M_{\odot} \mathrm{yr}^{-1}$ is assumed to be constant $(b=1)$. The dashed line corresponds to the flux from the stellar wind with velocity $v_{0}$. The behaviour of the plots is described in the text.

the central star. Contreras et al. (1996) presented the radiocontinuum flux (at different wavelengths) from P Cyg obtained on two sets of observations, showing that this source has timevariable thermal emission. In 1994, they measured the flux densities $S_{6 \mathrm{~cm}}=5.65 \pm 0.05 \mathrm{mJy}, S_{3.5 \mathrm{~cm}}=9.41 \pm 0.04 \mathrm{mJy}$ and $S_{0.7 \mathrm{~cm}}=27.4 \pm 1.2 \mathrm{mJy}$. For the 1995 observations, they detected $S_{6 \mathrm{~cm}}=9.13 \pm 0.07 \mathrm{mJy}, S_{3.5 \mathrm{~cm}}=12.18 \pm 0.04 \mathrm{mJy}$, $S_{2 \mathrm{~cm}}=16.6 \pm 0.2 \mathrm{mJy}$ and $S_{0.7 \mathrm{~cm}}=33.8 \pm 0.5 \mathrm{mJy}$. The spectra are shown in Figs. 5 and 6. Given the small uncertainties in the observations, the error bars are smaller than the symbols used in the plots.

In this section, we apply our model (described in Sect. 2) to both sets of observations. We investigate the possibility that the observed variability in the flux densities from P Cyg can be explained by a stellar wind subject to variations in the injection flow parameters. As mention in Sect. 2, time-dependent wind parameters (ejection velocity and mass loss rate) result in the formation of a working surface (two-shock wave structure) which travel down the wind. The contribution of the working surface to the total free-free emission depends on time (see Eqs. (2), (8), (14), (15)), and may be responsible for the observed variations in the flux densities. We assume the simple scenario of an isotropic wind with an internal working surface (moving with constant velocity; Eq. (1)) to study if our model is able to explain both set of observations by Contreras et al. (1996). We are therefore attempting to explain not only the clear deviation of the observed fluxes from the standard model $\left(S_{v} \propto v^{0.6}\right)$, but also the variability detected of the free-free emission. To this purpose we proceed as follows.

First, we fit independently the observed fluxes in 1994 and 1995. We adopt wind parameters consistent with observations of P Cyg, $v_{0}=220 \mathrm{~km} \mathrm{~s}^{-1}$ and $\dot{m}_{0}=0.33 \times 10^{-5} M_{\odot} \mathrm{yr}^{-1}$ which suddenly change to $286 \mathrm{~km} \mathrm{~s}^{-1}(a=1.3)$ and $10^{-5} M_{\odot} \mathrm{yr}^{-1}$ $(b=3.0)$, respectively. We find that our model is able to match the 1994 observations adopting $v_{\mathrm{c}}=10^{9} \mathrm{~Hz}$ and $f_{\mathrm{c}}=0.492 \mathrm{mJy}$, 


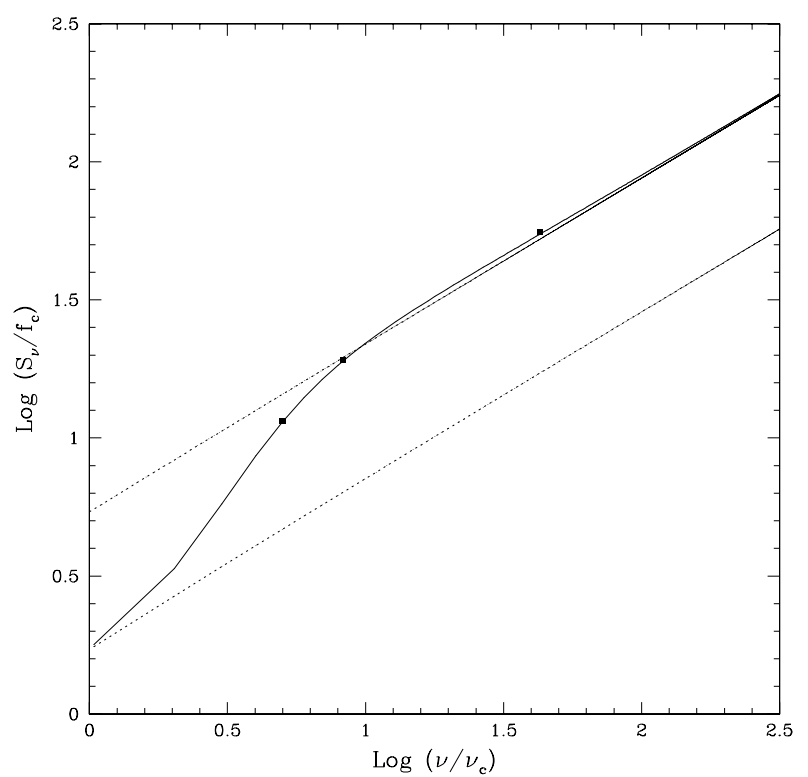

Fig. 5. Radio-continuum flux density from the P Cyg stellar wind as a function of frequency. For the model, we have assumed initial wind parameters $v_{0}=220 \mathrm{~km} \mathrm{~s}^{-1}$ and $\dot{m}_{0}=0.33 \times 10^{-5} M_{\odot} \mathrm{yr}^{-1}$, which suddenly increase to $286 \mathrm{~km} \mathrm{~s}^{-1}(a=1.3)$ and $10^{-5} M_{\odot} \mathrm{yr}^{-1}(b=3)$, respectively. The dashed lines represent the predicted spectrum $\left(\propto v^{0.6}\right)$ from the stellar winds $\left(v_{0}, \dot{m}_{0}\right.$ : lower line; $a v_{0}, b \dot{m}_{0}$ : upper line). The observed fluxes in 1994 (squares) by Contreras et al. (1996) are well fitted with $v_{\mathrm{c}}=10^{9} \mathrm{~Hz}$ and $f_{\mathrm{c}}=0.492 \mathrm{mJy}$. Given the small uncertainties in the observations (see the text), no error bars are shown in the figure.

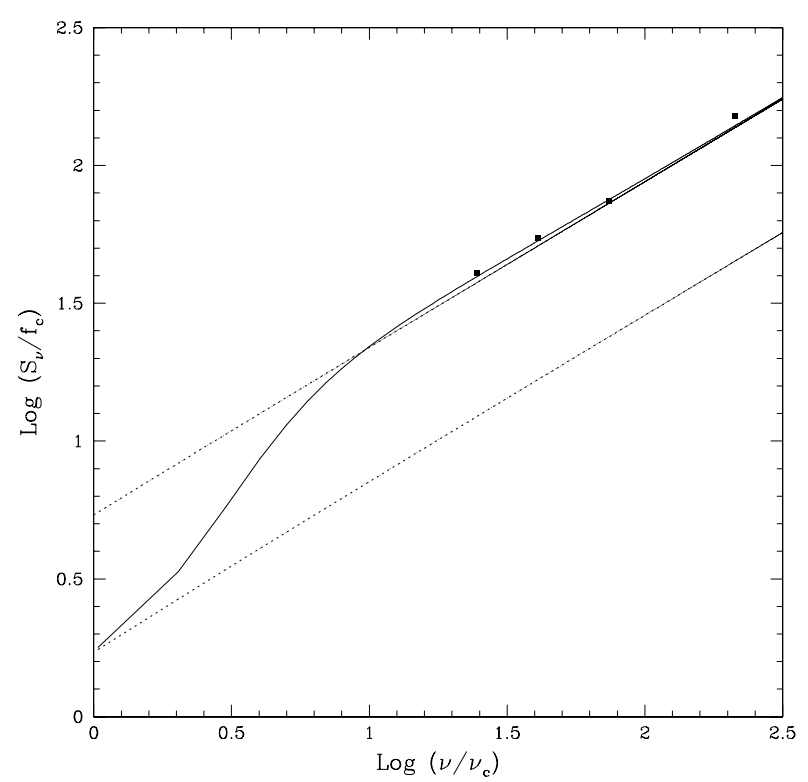

Fig. 6. The same as Fig. 5, but with the 1995 observations (squares) by Contreras et al. (1996) fit with $v_{\mathrm{c}}=2.03 \times 10^{8} \mathrm{~Hz}$ and $f_{\mathrm{c}}=0.224 \mathrm{mJy}$. Given the small uncertainties in the observations (see the text), no error bars are shown in the figure.

and a good fit of the 1995 observations is obtained with $v_{\mathrm{c}}=$ $2.03 \times 10^{8} \mathrm{~Hz}$ and $f_{\mathrm{c}}=0.224 \mathrm{mJy}$ (Figs. 5 and 6 ). However, such a variation in both parameters $v_{\mathrm{c}}$ and $f_{\mathrm{c}}$ does not occur over a period of time of $\sim 1 \mathrm{yr}$. In order to get a model that could explain the variability observed in P Cyg by Contreras et al. (1996) between 1994 and 1995, we insert the above values of $v_{\mathrm{c}}$ and $f_{\mathrm{c}}$
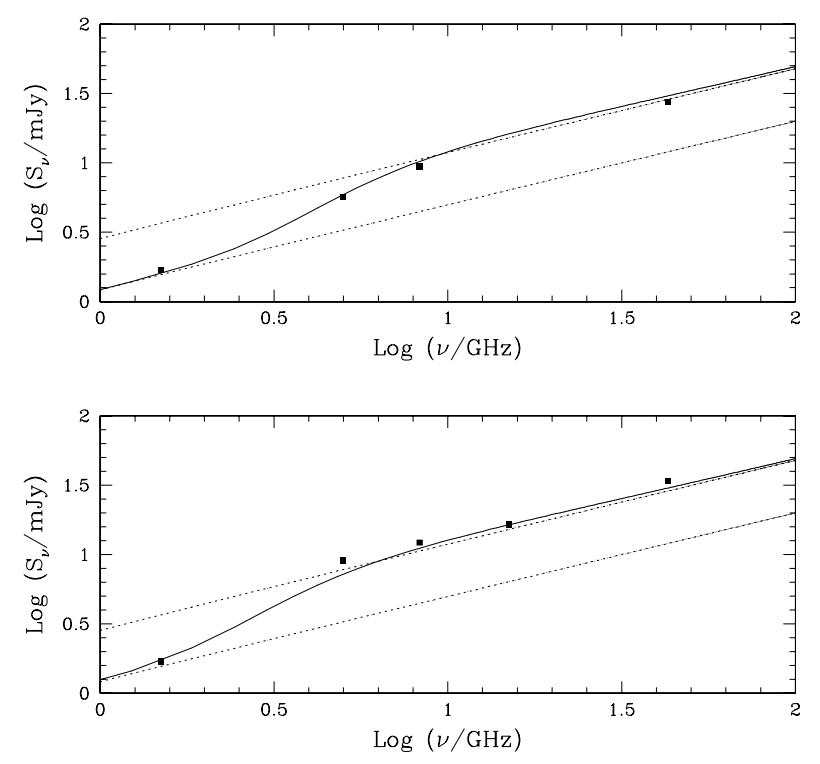

Fig. 7. Comparison between our model and the flux densities obtained in 1994 (top panel) and 1995 (bottom panel) by Contreras et al. (1996) from the P Cyg stellar wind. We have assumed $v_{0}=200 \mathrm{~km} \mathrm{~s}^{-1}$ and $\dot{m}_{0}=0.43 \times 10^{-5} M_{\odot} \mathrm{yr}^{-1}$, which suddenly change to $260 \mathrm{~km} \mathrm{~s}^{-1}$ ( $a=1.3$ ) and $1.075 \times 10^{-5} M_{\odot} \mathrm{yr}^{-1}(b=2.5)$, respectively. In the top panel, we present the spectrum at a time $t=3.3 \mathrm{yr}$ since the formation of the working surface and the observed 1994 flux densities (squares) from P Cyg. Bottom panel shows the predicted flux density after one year (at $t=4.3 \mathrm{yr}$ ) and the 1995 observations (squares). In both panels, we have also included a high angular resolution observation at $20 \mathrm{~cm}$ $(v=1.5 \mathrm{GHz})$ obtained from the VLA archives. The observation was taken in 1992, and it has been included in the plots under the justifiable assumption that the working surface had not affected yet the emission at this frequency. The dashed lines represent the predicted spectrum $\left(\propto v^{0.6}\right)$ from the stellar winds $\left(v_{0}, \dot{m}_{0}\right.$ : lower line; $a v_{0}, b \dot{m}_{0}$ : upper line). Given the small uncertainties in the observations (see the text), no error bars are shown in the figure. The physical description of the plot is given in the text.

into Eqs. (8) and (15), and then we obtain the following equations,

$$
\begin{aligned}
& 9.189 \times 10^{-3}-\dot{m}_{-5}^{0.95} v_{0,100}^{-2.38} t_{\mathrm{yr}}^{-1.43}=0 \\
& 1.865 \times 10^{-3}-\dot{m}_{-5}^{0.95} v_{0,100}^{-2.38}\left(t_{\mathrm{yr}}+1\right)^{-1.43}=0 \\
& 4.831 \times 10^{-3}-\dot{m}_{-5}^{1.90} v_{0,100}^{-2.76} t_{\mathrm{yr}}^{-0.86}=0 \\
& 2.244 \times 10^{-3}-\dot{m}_{-5}^{1.90} v_{0,100}^{-2.76}\left(t_{\mathrm{yr}}+1\right)^{-0.86}=0
\end{aligned}
$$

where $\dot{m}_{-5}$ is the mass loss rate in units of $10^{-5} M_{\odot} \mathrm{yr}^{-1}, v_{0,100}$ is the initial wind velocity in units of $100 \mathrm{~km} \mathrm{~s}^{-1}$, and $t_{\mathrm{yr}}$ is the time in units of years. Then, we search the minimim of the sum of the squares of Eqs. (16)-(19) as a function of several variables with expected values $\dot{m}_{-5}=0.33, v_{0,100}=2.2$ (which are the wind parameters used in Figs. 5 and 6) and $t_{\mathrm{yr}}=1$.

We find that the model (Eq. (14)) which matches better the flux densities of both 1994 and 1995 observations corresponds to $v_{0}=200 \mathrm{~km} \mathrm{~s}^{-1}$ and $\dot{m}_{0}=0.43 \times 10^{-5} M_{\odot} \mathrm{yr}^{-1}$, which increases to $260 \mathrm{~km} \mathrm{~s}^{-1}(a=1.3)$ and $1.075 \times 10^{-5} M_{\odot} \mathrm{yr}^{-1}$ $(b=2.5)$, respectively. In Fig. 7, we show the predicted spectrum by the model at a time $t=3.3 \mathrm{yr}$ (top panel) and $t=$ $4.3 \mathrm{yr}$ (bottom panel) since the formation of the working surface. Observational data of both epochs from P Cyg by Contreras et al. (1996) are represented by the squares. To test the validity of our model at lower frequencies, we searched in the VLA archives for high angular resolution observations made at $20 \mathrm{~cm}$ 
$(v=1.5 \mathrm{GHz})$. We found only one observation with these characteristics, taken in 1992 October 22. The total flux density of P Cyg was $1.69 \pm 0.21$ mJy (L.F. Rodríguez; private communication). The archive data were reduced following the standard procedures of the Very Large Array (VLA) of the National Radio Astronomy Observatory ${ }^{1}$. Even when this epoch is anterior to those when the higher frequency observations were made, we will include it in the comparison under the justifiable assumption that the working surface had not affected yet the emission at $20 \mathrm{~cm}$. After $t=3.3 \mathrm{yr}$ of expansion of the spherical shell (with $\left.v_{\mathrm{ws}} \simeq 235 \mathrm{~km} \mathrm{~s}^{-1}\right), r_{\mathrm{ws}}=2.4 \times 10^{15} \mathrm{~cm}($ Eq. (2)) from the central star. One year later (at $t=4.3 \mathrm{yr}$ ), the working surface is located at $r_{\mathrm{ws}}=3.2 \times 10^{15} \mathrm{~cm}$. From the comparison of the model with the 1994 observations, it is clear that our predictions are consistent with the significant departure (at low frequencies) in the spectral index from the expected 0.6 value. It is also remarkable that, even some observational data fall slightly outside the theoretical spectrum (this might be explained by the presence of more working surfaces given the short time-scale of variability in the P Cyg wind), our model is able to explain the increase in the flux densities (at all wavelengths) in a period of a year. This suggests that probably the changes detected in the free-free radio emission from P Cyg could be attributed to variations in the parameters of the wind at injection, resulting in the formation of internal shock waves.

Contreras et al. (2004) present a new set of radio-continuum observations (obtained in 1999) from P Cyg stellar wind. Comparing the new flux densities with previous ones, they found that they have decreased (by $\sim 70 \%, \sim 35 \%$, and $\sim 34 \%$ at $7 \mathrm{~mm}$, $3.6 \mathrm{~cm}$, and $6 \mathrm{~cm}$, respectively) from their 1995 values. They measured $S_{6 \mathrm{~cm}}=6.0 \pm 0.1 \mathrm{mJy}, S_{3.6 \mathrm{~cm}}=8.0 \pm 0.2 \mathrm{mJy}$ and $S_{0.7 \mathrm{~cm}}=9.7 \pm 2.1 \mathrm{mJy}$. From the $7 \mathrm{~mm}$ flux density, they derived a lower mass loss rate $\left(\dot{m}_{0}=0.5 \pm 0.2 \times 10^{-5} M_{\odot} \mathrm{yr}^{-1}\right)$ than that $\left(\simeq 10^{-5} M_{\odot} \mathrm{yr}^{-1}\right)$ reported by Contreras et al. (1996). Consequently, different wind parameters are requeried to produce flux densities as those observed in 1999 (see Fig. 8). We have adopted an initial wind velocity $v_{0}=200 \mathrm{~km} \mathrm{~s}^{-1}$ with an increased factor $a=2.1\left(420 \mathrm{~km} \mathrm{~s}^{-1}\right)$. The mass loss rate $\dot{m}_{0}=0.2 \times 10^{-5} M_{\odot} \mathrm{yr}^{-1}$ is assumed to be constant. These parameters give a velocity of the working surface $v_{\mathrm{ws}} \simeq 290 \mathrm{~km} \mathrm{~s}^{-1}$ (Eq. (1)). Figure 8 shows a comparison between our theoretical spectrum with the flux densities observed in 1999 by Contreras et al. (2004). The spectrum is obtained at a time $t=3.7 \mathrm{yr}$ since the formation of the spherical shell (note that this assumption implies that some drastic variations in the wind parameters may occur shortly after the 1995 observations). At this time, the working surface is located at a distance $r_{\mathrm{ws}}=3.37 \times 10^{15} \mathrm{~cm}$ from the central star. Clearly, the model represents quite well the observational data using reasonable flow parameters for the P Cyg stellar wind.

\section{Summary and conclusions}

In this paper, we develop a model for calculating the free-free radiation (at radio wavelengths) from the P Cyg wind. This source is very strong with a radio-continuum spectrum consistent with thermal emission, but with spectral indices that clearly deviate from the expected 0.6 value for a completely ionized isotropic wind. Our model proposes that there must be a contribution to the emission by compressed material (also photoionized by the

1 The National Radio Astronomy Observatory is a facility of the National Science Foundation operated under cooperative agreement by Associated Universities, Inc.

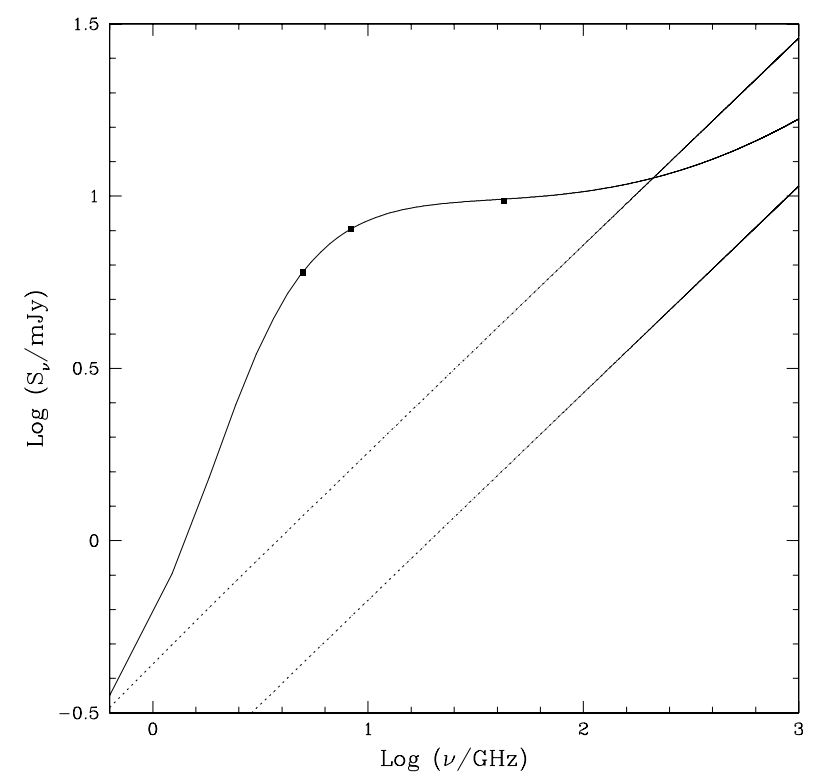

Fig. 8. Comparison between our model for the radio-continuum emission from the P Cyg wind and the 1999 observed flux densities (squares) by Contreras et al. (2004). We have assumed $v_{0}=200 \mathrm{~km} \mathrm{~s}^{-1}$ which suddenly increases to $420 \mathrm{~km} \mathrm{~s}^{-1}(a=2.1)$, and a constant $(b=1)$ mass loss rate $\dot{m}_{0}=0.2 \times 10^{-5} M_{\odot} \mathrm{yr}^{-1}$. We present the spectrum at a time $t=3.7 \mathrm{yr}$ since the formation of the working surface. The dashed lines represent the predicted spectrum $\left(\propto v^{0.6}\right)$ from the stellar winds $\left(v_{0}, \dot{m}\right.$ : upper line; $a v_{0}, b \dot{m}$ : lower line). Given the small uncertainties in the observations (see the text), no error bars are shown in the figure. The physical description of the plot is given in the text.

central star) between two-shock wave structures which result from varibilities in the wind parameters at injection. In particular, we assume sudden variations both in the wind velocity and the mass loss rate of the P Cyg wind which result in the formation of a compressed layer (bounded by two shock fronts) that generate thermal continuum radiation detectable at radio wavelengths. Assuming a colliding wind scenario (where a fast upstream wind interacts with a low-velocity downstream flow), we calculate the dynamical properties of the spherical shell and compute its optical depth. We obtain the total optical depth along each line of sight (taking into account the impact parameters that intersect the spherical shell) and then, we estimate the intensity emerging from each direction. Finally, we calculate the flux density by integrating the intensity over the solid angle.

Assuming a set of wind parameters consistent with observations of $\mathrm{P}$ Cyg, we find that our model can reproduce in a satisfactory way the detected radio-continuum fluxes of 1994 and 1995 by Contreras et al. (1996). Although some observed flux densities fall slightly outside of the theoretical spectrum, we show that the dynamical evolution of the compressed layer could probably explain the observed variability in the emission (on these two sets of observations) from P Cyg. Observations by Contreras et al. (2004) in 1999 give more evidence that P Cyg is a variable source. They show that the P Cyg fluxes had a considerable decrease from the 1995 values, suggesting a lower mass loss rate. Consequently, we adopted different flow parameters than those we use for the previous observations (in 1994 and 1995), and obtain that our results match the flux densities at different frequencies reported in Contreras et al. (2004). Since our model seems to fit the observed behaviour (at different epochs) of the flux densities and spectral indices from P Cyg, the radio-continuum emission from a fully ionized stellar wind with 
compressed material by shocks (due to time-dependent wind parameters) represents a possible scenario for explaining the observed variability from the P Cyg wind.

Acknowledgements. This work was supported by the CONACyT grant 46828-F, and the DGAPA (UNAM) grants IN 111606 and IN 113605. The authors have benefited from elucidating conversations and comments from L.F. Rodríguez which improved the content and presentation of the paper. The authors acknowledge Rosa Amelia González and an anonymous referee for their helpful comments.

\section{References}

Abbott, D. C., Bieging, J. H., \& Churchwell, E. 1981, ApJ, 250, 645 Cantó, J., Raga, A. \& D’Alessio, P. 2000, MNRAS, 313, 656

Cantó, J., González, R. F., Raga, A. C., et al. 2005, MNRAS, 357, 572

Contreras, M. E., Rodríguez, L. F., Gómez, Y., \& Velázquez, A. 1996, ApJ, 469, 329
Contreras, M. E., Montes, G., \& Wilkin, F. P. 2004, RevMexAA, 40, 53

Dyson, J. E., \& Williams, D. A. 1980, Physics of the Interstellar Medium (John Wiley \& Sons), 129

Exter, K. M., Watson, S. K., Barlow, M. J., \& Davis, R. J. 2002, MNRAS, 333, 715

Ghavamian, P., \& Hartigan, P. 1998, ApJ, 501, 687

González, R. F., \& Cantó, J. 2002, ApJ, 580, 459

González, R. F., Montes, G., Cantó, J., \& Loinard, L. 2006, MNRAS, 373, 391

Humphreys, R. M., Davidson, K., \& Smith, N. 1999, PASP, 111, 1124

Najarro, F., Hillier, D. J., \& Stahl, O. 1997, A\&A, 326, 1117

Panagia, N., \& Felli, M. 1975, A\&A, 39, 1

Raga, A. C., Cantó, J., Binette, L., \& Calvet, N. 1990, ApJ, 364, 601

Scuderi, S., Bonanno, G., Spadaro, D., et al. 1994, ApJ, 437, 465

Skinner, C. J., Becker, R. H., White, R. L., et al. 1998, MNRAS, 296, 669

Smith, N., \& Hartigan, P. 2006, ApJ, 638, 1045

van den Oord, G. H. J., Waters, L. B. F., Lamers, H. J. G. L. M., et al. 1985, in Radio Stars, ed. R. M. Hjellming, \& D. M. Gobson, 111

Wright, A. E., \& Barlow, M. J. 1975, MNRAS, 170, 41 